\title{
Phenomenology of Dynamical Dark Matter
}

\author{
Keith R. Dienes \\ Physics Division, National Science Foundation, Arlington, VA 22230, USA \\ Department of Physics, University of Maryland, College Park, MD 20742, USA \\ Department of Physics, University of Arizona, Tucson, AZ 85721, USA \\ E-mail: dienes@physics.arizona.edu
}

\section{Brooks Thomas*}

Department of Physics, University of Hawaii, Honolulu, HI 96822 USA

E-mail: thomasbd@phys.hawaii.edu

\begin{abstract}
Dynamical dark matter (DDM) is a new framework for dark-matter physics in which the dark sector comprises an ensemble of individual constituent fields, and in which the usual requirement of dark-matter stability is replaced by a balancing between constituent lifetimes and cosmological abundances across the ensemble as a whole. In this article, we review some of the phenomenological possibilities inherent within the DDM framework. In particular, we discuss some of the ways in which DDM ensembles can be distinguished from traditional dark-matter candidates experimentally, both at the LHC and at the next generation of direct-detection experiments.
\end{abstract}

36th International Conference on High Energy Physics

4-11 July 2012

Melbourne, Australia

\footnotetext{
* Speaker.
} 


\section{Introduction}

Dynamical dark matter (DDM) [1], 2] is a new framework for dark-matter physics in which the dark sector comprises an ensemble of individual constituent fields, and in which the usual requirement of dark-matter stability is replaced by a balancing between constituent lifetimes and cosmological abundances across the ensemble as a whole. Such DDM ensembles have highly nontrivial cosmological consequences, including a constantly evolving composition of the dark-matter relic abundance and a time-dependent dark-matter equation of state [价. In addition, these ensembles also give rise to characteristic phenomenological signatures which can differ significantly from those associated with more traditional dark-matter candidates. In this article, we review some of the ways in which DDM ensembles can be distinguished from traditional dark-matter candidates experimentally, both at the LHC [4] and at the next generation of direct-detection experiments [5].

\section{Distinguishing DDM at the LHC}

One promising technique for distinguishing DDM ensembles is to examine the kinematic distributions of visible particles produced in conjunction with the constituents via the decays of other, heavier "parent" particles [ [ [ at the LHC. As an example of how this technique can be applied, let us consider a concrete example in which the constituent fields $\chi_{n}$ of the DDM ensemble are gauge-singlet fermions, and in which the theory also contains a heavy color-octet fermion $\psi$, which couples to the $\chi_{n}$ via an effective operator of the form

$$
\mathscr{L}_{\text {eff }}=\sum_{n}\left[\frac{c_{n}}{\Lambda^{2}}\left(\bar{q}_{i} t_{i j}^{a} \psi^{a}\right)\left(\bar{\chi}_{n} q_{j}\right)+\text { h.c. }\right]
$$

Here $q_{i}$ denotes a SM quark, $c_{n}$ is a dimensionless operator coefficient, $\Lambda$ is the cutoff scale of the effective theory, $t_{i j}^{a}$ is the generator of $S U(3)$ in the fundamental representation, and $i$ and $j$ are color indices. This operator gives rise to decays of the form $\psi \rightarrow j j \chi_{n}$, where $j$ denotes a hadronic jet. As we shall see, the invariant-mass distribution for the jets produced in these decays can yield important information about the properties of the dark-matter candidate.

We now examine more explicitly how the invariant-mass distributions associated with DDM ensembles differ from those associated with traditional dark-matter candidates. For concreteness, we choose to work in the context of a simplified DDM model in which the mass spectrum of the $\chi_{n}$ is assumed to take the form

$$
m_{n}=m_{0}+n^{\delta} \Delta m
$$

with $\Delta m>0$ and $\delta>0$, so that the $\chi_{n}$ are labeled in order of increasing mass. In addition, we assume that the operator coefficients across the ensemble exhibit a power-law scaling of the form

$$
c_{n}=c_{0}\left(\frac{m_{n}}{m_{0}}\right)^{\gamma}
$$

where $\gamma$ is general power-law exponent. We also assume that the the $\chi_{n}$ which are kinematically accessible in $\psi$ are stable on collider time scales. In Fig. 1, we display a set of invariant-mass distributions which arise within the parameter space of our simplified DDM model. Comparing the different curves shown in each panel, we see that as $\gamma$ increases and the couplings of the heavier $\chi_{n}$ 

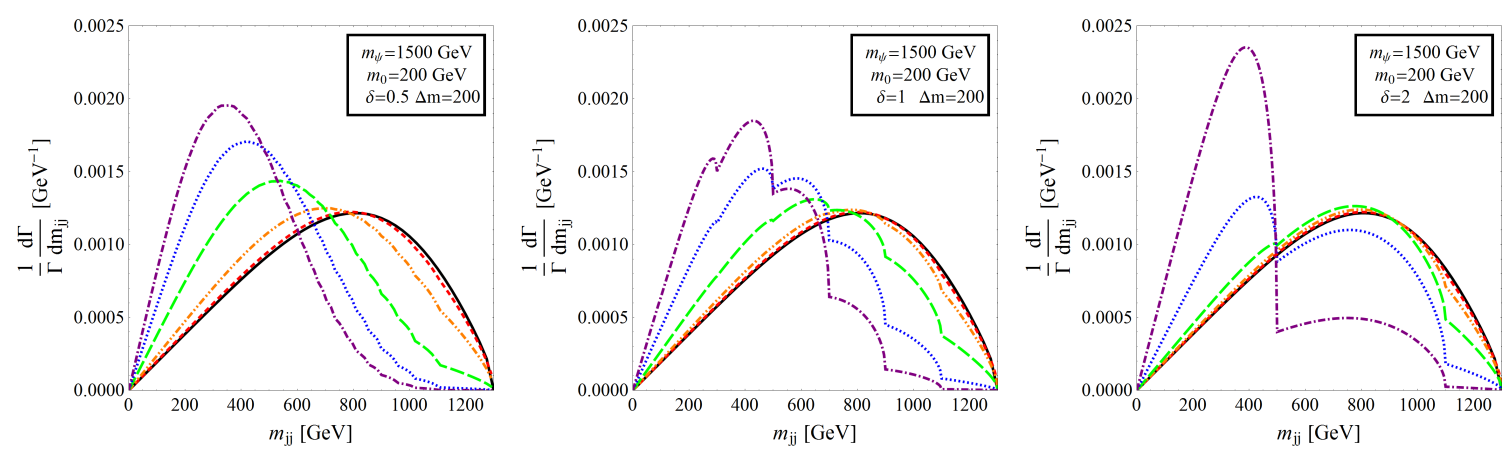

Figure 1: Invariant-mass distributions demonstrating how a DDM ensemble can be distinguished from traditional dark-matter candidates. In each panel, the red, orange, green, blue, and purple curves correspond to $\gamma=\{-2,-1,0,1,2\}$, respectively, while the black curve shows the result for a traditional dark-matter candidate with $m_{\chi}=m_{0}$.

become significant, two characteristic features emerge within different regimes. For large $\delta$ (and/or $\Delta m$ ), multiple distinct mass edges can be resolved within the overall $m_{j j}$ distribution. By contrast, for sufficiently small $\delta$ (and/or $\Delta m$ ), these mass edges are not readily distinguishable; rather, the invariant-mass distribution assumes a characteristic shape, with no evident mass edge and with a peak shifted to lower values of $m_{j j}$. Both of these features can serve to distinguish DDM ensembles from traditional dark-matter candidates at colliders, and in particular at the LHC.

In order to quantitatively assess the degree to which the invariant-mass spectrum associated with a given DDM ensemble can be distinguished from that associated with a traditional darkmatter candidate, we adopt the following procedure. (For details, see Ref. [⿰亻⿻乚㇒日.) We partition the two distributions into bins with widths given by the invariant-mass resolution of the detector and assess the goodness of fit between the two distributions by constructing a $\chi^{2}$ statistic. We then survey over $m_{\chi}$ and take the minimum $\chi^{2}$ value obtained in this survey to be our final measure of the distinctiveness of the $m_{j j}$ distribution associated with the DDM ensemble. In Fig. Z, we display contours of the statistical significance of differentiation obtained in our simplified DDM model for $N_{e}=1000$ signal events - roughly the event count expected from the pair-production of a $\psi$ with a TeV-scale mass at the $\sqrt{s}=14 \mathrm{TeV}$ LHC with $\mathscr{L}_{\text {int }} \approx 30 \mathrm{fb}^{-1}$. Note that a high significance of differentiation is typically obtained in regions of parameter space within which $\gamma>0$ and $\delta$ and $\Delta m$ are both reasonably small. Indeed, these are the regions of parameter space within which the characteristic features depicted in Fig. 11 are particularly pronounced. As $\delta$ and $\Delta m$ increase, the significance of differentiation remains small even for large $\gamma$, save for a narrow strip of parameter space within which the mass edges associated with both $\chi_{0}$ and $\chi_{1}$ - the only DDM constituents kinematically accessible in $\psi$ decays for large $\delta$ and $\Delta m$ - can be independently resolved. The results shown in Fig. 目demonstrate that kinematic distributions can play an important role in distinguishing DDM ensembles from traditional dark-matter candidates at the LHC.

\section{Distinguishing DDM at Direct-Detection Experiments}

Direct-detection experiments offer another possible method for distinguishing DDM ensembles [5]. Indeed, characteristic features in the recoil-energy spectra observed at these experiments 

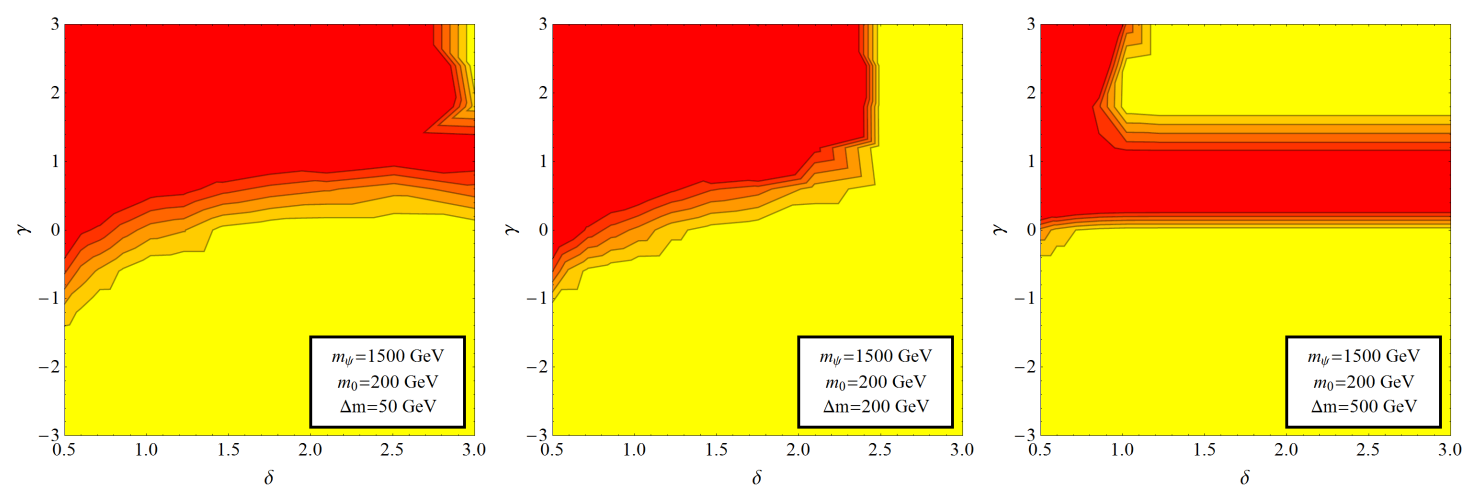

Significance :
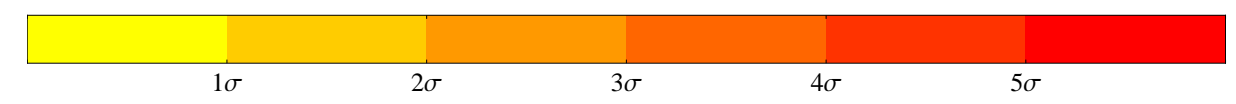

Figure 2: Contour plots showing the minimum significance level at which the $m_{j j}$ distribution predicted in our simplified DDM model can be differentiated from the $m_{j j}$ distribution predicted in any traditional dark-matter model. In each panel we have assumed a sample size of $N_{e}=1000$ events.

can serve as a basis for distinguishing such dark-matter candidates from their traditional counterparts. For simplicity, we focus on the case in which elastic scattering dominates the scattering rate for all $\chi_{j}$. In this case, the total differential scattering rate is simply the sum of the scattering rates associated with each individual $\chi_{j}$ :

$$
\frac{d R}{d E_{R}}=\sum_{j} \frac{\sigma_{N j}^{(0)} \rho_{j}^{\text {loc }}}{2 m_{j} \mu_{N j}^{2}} F^{2}\left(E_{R}\right) I_{j}\left(E_{R}\right),
$$

where $E_{R}$ is the recoil energy of the scattered nucleus in the detector frame, $\sigma_{N j}^{(0)}$ is the $\chi_{j}$-nucleus scattering cross-section at zero momentum transfer, $\rho_{j}^{\text {loc }}$ is the local energy density of $\chi_{j}, F\left(E_{R}\right)$ is a nuclear form factor, $m_{N}$ is the mass of the scattered nucleus $N, m_{j}$ is the mass of $\chi_{j}, \mu_{N j} \equiv$ $m_{\chi} m_{N} /\left(m_{j}+m_{N}\right)$ is the reduced mass of the $\chi_{j}$-nucleus system, and $I_{\chi}\left(E_{R}\right)$ is the mean inverse speed of $\chi_{j}$ in the dark-matter halo for a given $E_{R}$.

As with the collider analysis presented in Sect. 2, we examine the direct-detection phenomenology of DDM ensembles within the context of a simplified DDM model. In this model, the mass spectrum is taken to have the same form as in Eq. (2.2), and the present-day relic abundances $\Omega_{j}$ and the effective $\chi_{j}$-nucleon coupling coefficients $f_{n j}$ each exhibit a power-law scaling of the form

$$
\Omega_{j}=\Omega_{0}\left(\frac{m_{j}}{m_{0}}\right)^{\alpha}, \quad f_{n j}=f_{n 0}\left(\frac{m_{j}}{m_{0}}\right)^{\beta},
$$

with respective power-law exponents $\alpha$ and $\beta$. Moreover, for concreteness, we adopt a benchmark set of standard assumptions about the additional particle-physics, astrophysics, and nuclear-physics considerations which impact the differential recoil rate. The interactions of each $\chi_{j}$ are taken to be isospin-conserving and isotropic. The total local dark-matter density, is taken to be $\rho_{\text {tot }}^{\text {loc }} \approx$ $0.3 \mathrm{GeV} / \mathrm{cm}^{3}$, and we assume that $\rho_{j}^{\text {loc }} / \rho_{\text {tot }}^{\text {loc }}=\Omega_{j} / \Omega_{\text {tot }}$ for all $\chi_{j}$. The velocity distribution of particles in the dark-matter halo is taken to be Maxwellian, and the local circular velocity and galactic escape velocity are taken to be $v_{0} \approx 220 \mathrm{~km} / \mathrm{s} v_{\mathrm{esc}} \approx 540 \mathrm{~km} / \mathrm{s}$, respectively. The nuclear 

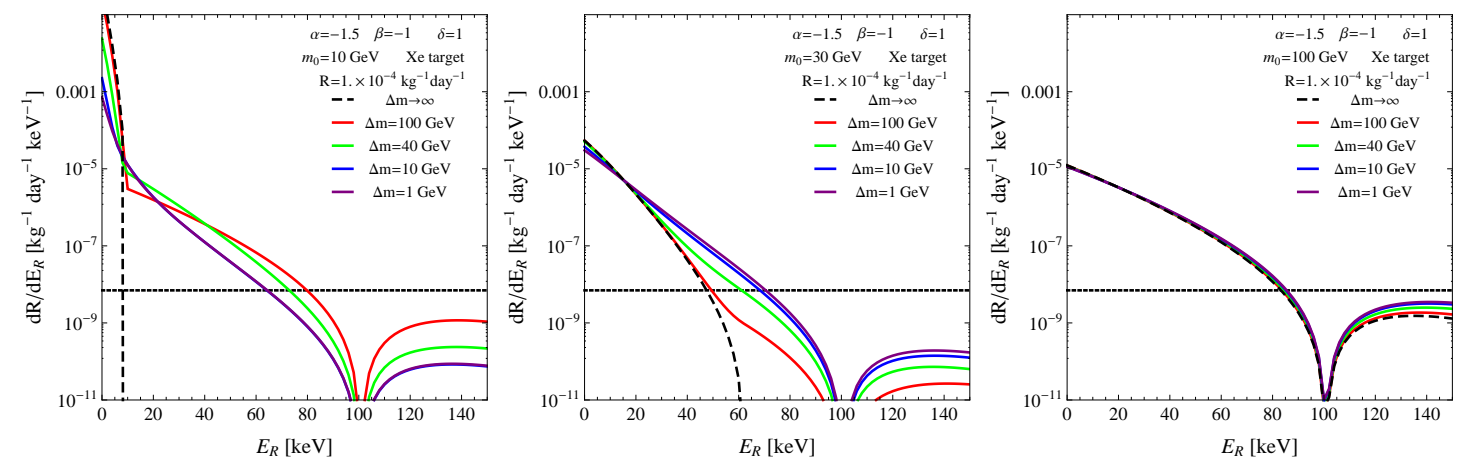

Figure 3: Recoil-energy spectra associated with DDM ensembles scattering elastically off of a xenon target. The different curves displayed in each panel correspond to different values of $\Delta m$. Note that the $\Delta m \rightarrow \infty$ limit indicated by the dashed black curve corresponds to a traditional dark-matter candidate with a mass $m_{\chi}=m_{0}$. The dotted black horizontal line indicates a reasonable estimate of the recoil-energy spectrum for background events at the next generation of liquid-xenon detectors.

form factor $F\left(E_{R}\right)$ is taken to have the Helm functional form. A variety of departures from this standard benchmark can have an impact on recoil-energy spectra. However, our aim here will be to focus on the effect of replacing a traditional dark-matter candidate with a DDM ensemble while holding all other aspects of this benchmark fixed.

In Fig. 月, we display the recoil-energy spectra obtained for our simplified DDM model for scattering off a xenon target for several different choices of model parameters. Note that for small values of $m_{0}$ and $\Delta m$ the contributions from multiple different $\chi_{j}$ in the "low-mass regime" $m_{j} \lesssim$ $20 \mathrm{GeV}$ conspire to produce a distinctive ogee-shaped curve. Similarly, in cases where $\Delta m$ is large and all of the $\chi_{j}$ with $j \geq 1$ lie within the opposite, "high-mass" regime $m_{j} \gtrsim 20 \mathrm{GeV}$, an equally distinctive kink appears in the recoil-energy spectrum. By contrast, when $m_{0}$ is large and all of the $\chi_{j}$ lie within the high-mass regime, the shapes of the recoil-energy spectra do not differ appreciably from those associated with traditional dark-matter candidates. This is because the individual contributions to the recoil-energy spectrum from $\chi_{j}$ in the high-mass regime do not depend sensitively on $m_{j}$.

In order to assess more quantitatively the potential for differentiating between DDM ensembles and traditional dark-matter candidates, we examine the statistical significance of differentiation at a hypothetical dual-phase xenon detector with attributes similar to those projected for XENON1T and future phases of the LUX experiment. (For more details, see Ref. [5].) For concreteness, we adopt a benchmark value of $N_{e}=1000$ total signal events after five live years of running. In Fig. $\sharp$, we display contours of the statistical significance of differentiation for our DDM ensemble in $\left(m_{0}, \Delta m\right)$ space. The results shown indicate that there are two primary regions in which a high statistical significance of differentiation is obtained. The first is the region where $\left\{m_{0}, \Delta m\right\} \lesssim$ $30 \mathrm{GeV}$, within which the characteristic ogee shape indicated in Fig. 3 arises for sufficiently small $\beta$. The second is the region where $5 \mathrm{GeV} \lesssim m_{0} \lesssim 20 \mathrm{GeV}$, but $\Delta m$ is somewhat larger, in which a discernible kink appears in the recoil-energy spectrum, as discussed above. 

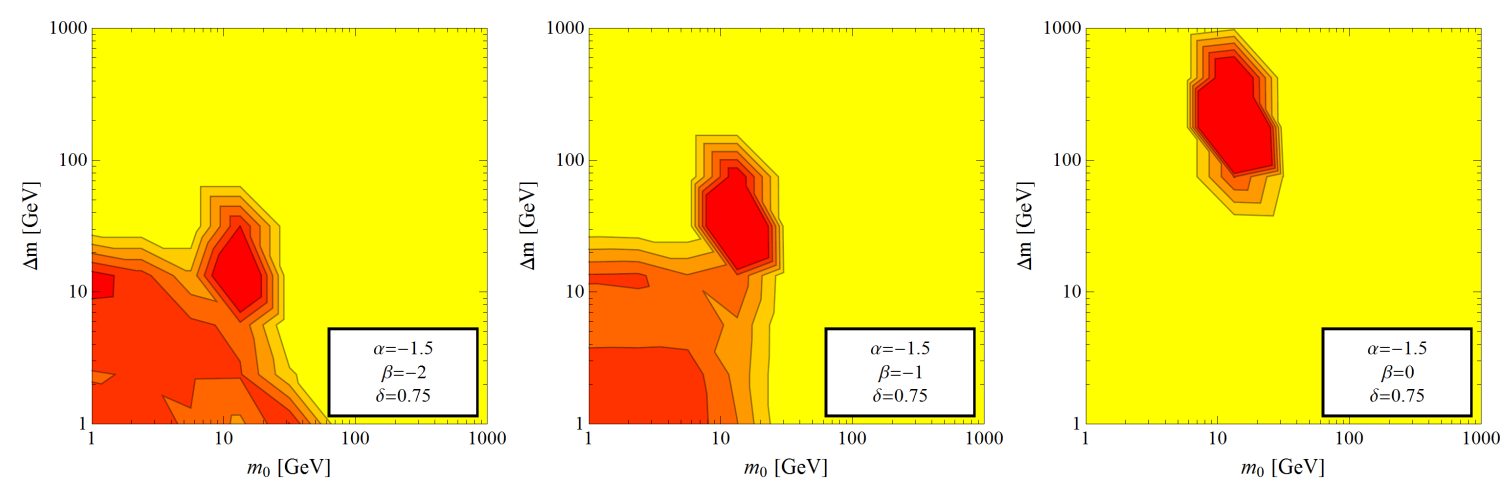

Figure 4: Contour plots showing the significance level at which the recoil-energy spectrum associated with a DDM ensemble can be distinguished from that associated with any traditional dark-matter candidate which gives rise to the same total event rate at our hypothetical next-generation direct-detection experiment. The colored regions shown correspond to the same significance intervals as in Fig. Z.

\section{Conclusions}

In this article, we have reviewed several characteristic signatures to which DDM ensembles give rise and examined the prospects for distinguishing these ensembles from traditional darkmatter candidates on the basis of such signatures. We have focused here on a particular set of signatures at the LHC and at the next generation of direct-detection experiments, but we emphasize that a wide variety of additional possibilities for experimentally distinguishing DDM ensembles remain to be explored.

\section{Acknowledgments}

KRD is supported in part by the U.S. Department of Energy under Grant No. DE-FG0204ER-41298 and by the National Science Foundation through its employee IR/D program. BT is supported in part by DOE Grant No. DE-FG02-04ER-41291. The opinions and conclusions expressed herein are those of the authors, and do not represent either the Department of Energy or the National Science Foundation.

\section{References}

[1] K. R. Dienes and B. Thomas, Phys. Rev. D 85, 083523 (2012) [arXiv:1106.4546 [hep-ph]].

[2] K. R. Dienes and B. Thomas, Phys. Rev. D 85, 083524 (2012) [arXiv:1107.0721 [hep-ph]].

[3] K. R. Dienes and B. Thomas, Phys. Rev. D 86, 055013 (2012) [arXiv:1203.1923 [hep-ph]].

[4] K. R. Dienes, S. Su and B. Thomas, Phys. Rev. D 86, 054008 (2012) [arXiv:1204.4183 [hep-ph]].

[5] K. R. Dienes, J. Kumar and B. Thomas, Phys. Rev. D 86, 055016 (2012) [arXiv:1208.0336 [hep-ph]]. 